The ASTROPHYSICAL JOURNAL SUPPLEMENT SERIES, 90:893-897, 1994 February

(c) 1994. The American Astronomical Society. All rights reserved. Printed in U.S.A.

\title{
DO GAMMA-RAY BURSTS ORIGINATE FROM AN EXTENDED GALACTIC HALO OF HIGH-VELOCITY NEUTRON STARS?
}

\author{
Dieter H. Hartmann, Lawrence E. Brown, and Lih-Sin The \\ Department of Physics and Astronomy, Clemson University, Clemson, SC 29634-1911 \\ ERIC V. LiNDER aND VAHÉ Petrosian \\ Center for Space Science and Astrophysics, Stanford University, Stanford, CA 94305 \\ George R. Blumenthal \\ UCO/Lick Observatory, University of California at Santa Cruz, Santa Cruz, CA 95064 \\ AND \\ KEVIN C. HURLEY \\ Space Sciences Laboratory, University of California at Berkeley, Berkeley CA 94720 \\ Received 1993 March 2; accepted 1993 May 27
}

\begin{abstract}
The $\gamma$-ray burst brightness distribution is inhomogeneous and the distribution on the sky is nearly isotropic. These features argue against an association of $\gamma$-ray bursts with those Galactic objects that are known to exhibit a strong concentration toward the Galactic center or plane. The observed statistical properties indicate a cosmological origin. Circumstantial evidence suggests that neutron stars are involved in the burst phenomenon. Here we consider Population II neutron stars in an extended Galactic Halo (EGH) as an alternative to cosmological scenarios. The BATSE data indicate a small deviation from isotropy near the $2 \sigma$ level of statistical significance. If confirmed for an increasing number of bursts, these anisotropies could rule out cosmological scenarios. On the other hand, EGH models require small anisotropies like those observed by BATSE. We consider simple distribution models to determine the generic properties such halos must have to be consistent with the observations and discuss the implications of the corresponding distance scale on burst models.
\end{abstract}

Subject headings: gamma rays: bursts — stars: neutron — stars: Population II — stars: statistics

\section{INTRODUCTION}

As of 1993 February 22, the BATSE experiment aboard Compton Observatory detected 576 cosmic $\gamma$-ray bursts (GRBs). The burster brightness distribution shows that the source distribution in space must be nonuniform, while the localizations suggest that their sky distribution is nearly isotropic (Meegan et al. 1992a, b). If the burster sky were indeed isotropic, cosmological models would provide the simplest framework for their origin (Paczyński 1991). Deviations from isotropy would severely constrain or perhaps even rule out a cosmological origin. Strong anisotropies expected from Galactic disk populations rule out the possibility that most bursters are associated with the Galactic disk, but a small "contamination" of an otherwise isotropic background is conceivable. Similar arguments apply to the nearly, but not perfectly, isotropic distribution expected from sources in an extended halo, although contamination by disk sources might be more natural in this context. Figure 1 shows the angular distribution of the first 260 BATSE events (Meegan et al. 1992c) in a fuzzy format, i.e., each individual burst is spread over the sky according to its localization accuracy ( $1 \sigma$ error radii) convolved (in quadrature) with a $4^{\circ}$ systematic error. It is clear, through inspection by eye, that any possible deviation of the BATSE burst locations from isotropy must have low statistical significance. Note the absence of obvious burst frequency enhancements along the directions to nearby galaxies, such as M31 and the
Magellanic clouds, and prominent galaxy clusters. The distribution is not corrected for the sky exposure map, but the statistical estimates of low-order multipoles presented below take the uneven sampling of the sky into account.

Halo distributions similar to that inferred for the Galactic dark matter corona do not yield sky distributions that have the required statistical properties (Paczyński 1991; Hakkila et al. 1992; Mao \& Paczyński 1992), but it was argued (Brainerd 1992; Eichler \& Silk 1992; Hartmann et al. 1992; Hartmann 1992 ) that neutron stars in an extended Galactic halo could satisfy all observational constraints, if one considers Population II stars. An extended halo created by injection of high-velocity neutron stars from the Galactic plane (Shklovskii \& Mitrofanov 1985) causes unacceptably large anisotropies, unless early bursting activity is suppressed ( Li \& Dermer 1992). Injection from a halo distribution of parent bodies alleviates some of the problems associated with disk injection (Hartmann 1992; Fabian \& Podsiadlowski 1993). The recent discovery (Harrison, Lyne, \& Anderson 1993) of high-velocity radio pulsars well above the Galactic plane lends some support to the notion that neutron star formation in the halo could provide the GRB sources.

The extent of such a bursting halo population is constrained by the BATSE statistics. While coordinate independent techniques are superior for the task of detecting arbitrary anisotropies on the sky (Hartmann \& Epstein 1989), EGH models can be tested efficiently via low-order multipoles in galactic coordi- 


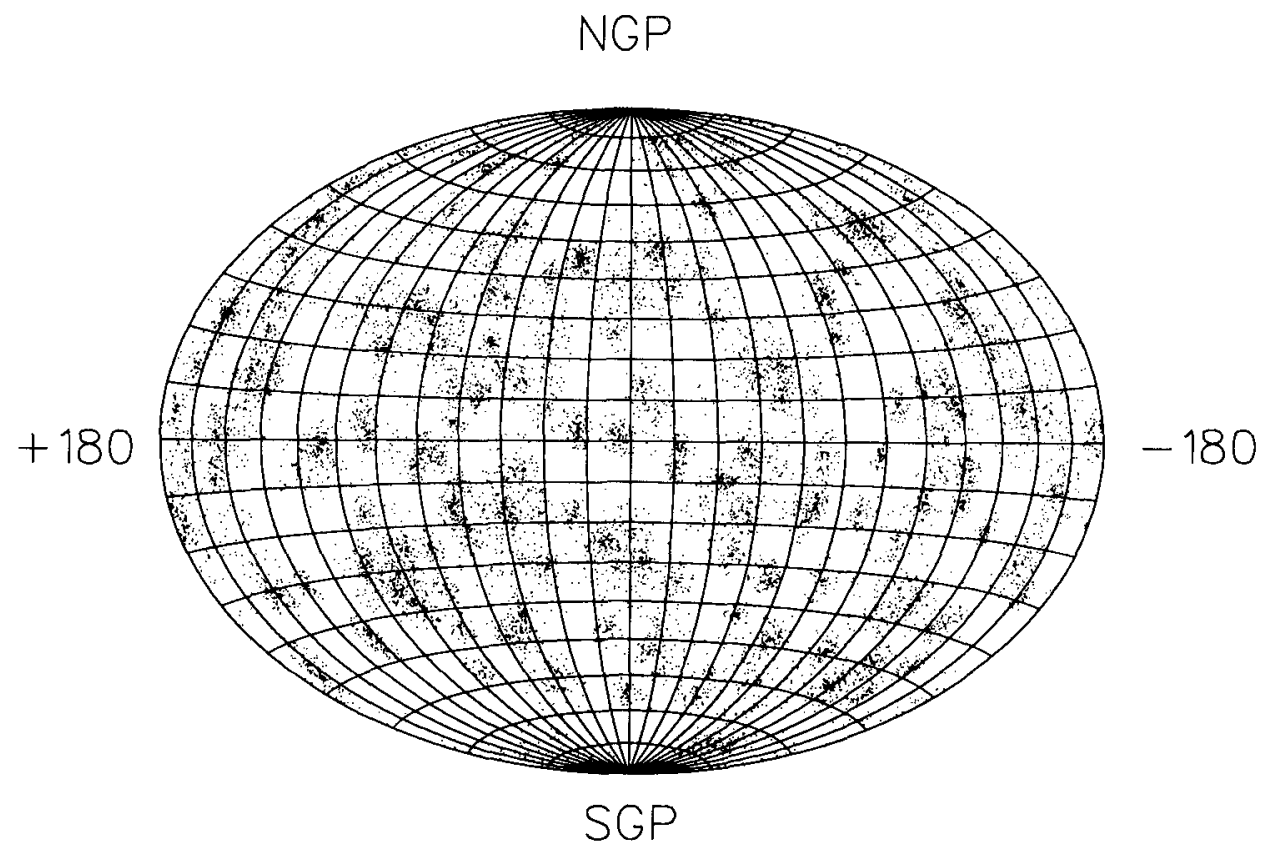

FIG. 1.-Fuzzy (see text) distribution of 260 BATSE bursts in Galactic coordinates

nates (Paczyński 1990). The BATSE statistics of 447 events (Meegan et al. 1992d) show a dipole moment of

$$
D=\langle\cos \theta\rangle=0.034 \pm 0.027,
$$

where the error is statistical only. The quadrupole moment is

$$
Q=\left\langle\sin ^{2}(b)\right\rangle-1 / 3=-0.017 \pm 0.014 \text {. }
$$

These values are very close to those expected from isotropy, $(D, Q)_{\text {iso }}=(0,0)$, so that small effects due to uneven sampling of the sky may become important. Therefore, the BATSE team spent great efforts to derive the sky exposure map for their instrument (Brock et al. 1992). Based on this map, a perfectly isotropic burst distribution would have resulted in the measured moments $(D, Q)_{\text {map }}=(-0.014,-0.004)$. Because errors in $(D, Q)$ due to the localization uncertainties of individual bursts are expected to be small (Horack et al. 1993) we can subtract the map moments from the observed moments to obtain an estimate of the intrinsic moments. Figure 2 shows the results; the data suggest anisotropies of the intrinsic burst distribution at the $2 \sigma$ level. While this may not be significant enough to rule out a cosmological origin of $\gamma$-ray bursts, we note the fact that the moments point in the direction expected from EGH models.

\section{NEUTRON STARS IN THE GALACTIC HALO}

The high degree of isotropy clearly requires a very extended halo of bursting objects. If one retains the neutron star paradigm, the crucial question is how to populate such an extended region and how to avoid the highly anisotropic contributions from neutron stars residing in the disk. A clue to the answer might come from a recent pulsar proper motion survey (Harrison et al. 1993). The radio data suggest a significant extension of neutron star space velocities over that infered from previous surveys. In several cases, velocities of $\sim 1000 \mathrm{~km} \mathrm{~s}^{-1}$ were detected. A significant fraction of pulsars in the high-velocity tail of the distribution are not gravitationally bound to the Galaxy. An interesting aspect of these observations is the presence of several high-velocity pulsars that appear to be traveling toward the Galactic plane, while most pulsars are known to migrate away from the plane. Some of these pulsars could be due to runaway OB-stars and some could be returning pulsars that were born in the plane with high velocities (these, however, must then be very old, in conflict with typical pulsar ages of $\sim 10^{7} \mathrm{yr}$ ). Furthermore, some of the returning pulsars have small characteristic ages. Although there are currently less than

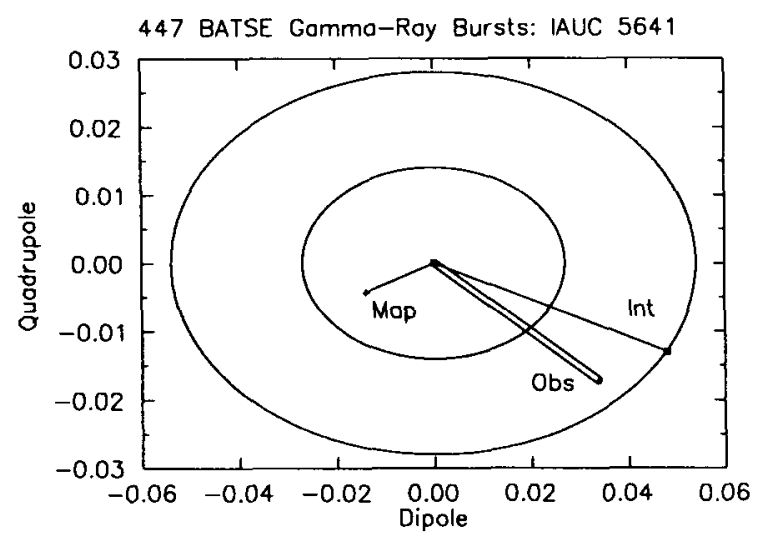

FIG. 2.-Galactic gamma-ray burst multipoles for 447 events observed with BATSE. The data suggest a $2 \sigma$ detection of sky distribution anisotropies. The intrinsic (Int) source moments are obtained from combining the observed values (Obs) with those expected for an isotropic distribution convolved with the non-uniform sky sensitivity map of BATSE. Shown are the 1 and $2 \sigma$ statistical error contours for 447 events. 
a handful of these pulsars, their observed properties cast some doubt on the traditionally held views about pulsar birthplaces. A possible interpretation of these observations is that neutron star formation is an ongoing process high above the Galactic plane (Hartmann 1992; Eichler \& Silk 1992). We show below that the formation of high-velocity neutron stars in an already extended halo produces a spatial distribution that satisfies the BATSE isotropy constraint. In contrast to injection from the disk, injection from the halo produces a much more isotropic distribution. If neutron star birth in the halo is due to the merger events of pregalactic white dwarf binaries (Eichler \& Silk 1992), nucleosynthesis during these events and the emission of neutrino bursts can be used to constrain the long-term average or recent merger rate, respectively (Hartmann 1992). From these constraints it is clear that the ratio of the number of neutron stars formed in the halo to that formed in the Galactic disk should be less than $\sim 10^{-3}$. We therefore have less than $10^{5}$ to $10^{6}$ sources available to produce the observed rate of $\sim 10^{3}$ bursts per year (Meegan et al. 1992a). This implies a recurrence time of less than 100-1000 years, which is consistent with current estimates of $\sim 10 \mathrm{yr}$. However, one of the major questions is why this small population produces such prolific bursters, while the large population of disk neutron stars appears to be $\gamma$-ray quiet. One possible answer to that question could be due to a correlation between the velocity of neutron stars and their emission directionality ( Li \& Dermer 1992; Duncan, Li, \& Thompson 1993). If burst emission is beamed preferentially along the direction of motion, as suggested by the "magnetars" model of Duncan \& Thompson (1992), then the detection probability increases with source distance from the galactic center, and the contamination problem can be avoided even if there are a larger number of events in the Galactic disk.

\section{ISOTROPY}

To determine the spatial distribution of neutron stars in an $\mathrm{EGH}$, one could directly calculate stellar orbits in a realistic Galactic potential, but at the birth sites and velocities relevant for the EGH models considered here the orbits are very close to straight lines. Isotropic injection at some location $r_{0}$ with velocity $v_{0}$ leads to expanding galactocentric shells of widths $\Delta R=$ $2 R_{\odot}$, with $R_{\odot}=8.5 \mathrm{kpc}$. The burster brightness distribution is a function of many (unknown) properties; radial distribution of birthplaces and birthdates, initial velocities, burst rate as a function of age, and the intrinsic luminosity function. Obviously, it would not be too hard to find a set of parameters that satisfies the observed $V / V_{\max }$ statistics. One has less freedom with the angular distribution. In the case of spherical, galactocentric shells, the observed anisotropies depend on the sampling depth from Earth and the radial "occupation probabilities" of the shells sampled. For simplicity, we ignore effects due to varying detector thresholds (Band 1992; Hartmann \& The 1992; Petrosian 1992) and the dependence on the (unknown) luminosity function, and consider the appearance of bursts uniformly located on shells of radius $x=R / R_{\odot}$. A single shell contributes a dipole moment

$$
D=\frac{2}{3} x^{-1}
$$

and a quadrupole moment

$$
Q=\frac{1}{8} x^{-1}\left\{x^{3}+x-\frac{1}{2}\left(1-x^{2}\right)^{2} \ln \left(\frac{1+x}{|x-1|}\right)\right\}-\frac{1}{3} .
$$

Obviously, the dipole component provides the most stringent constraints for this class of models, but because of the relationship between the two moments ( $Q=$ const $D^{2}$ for large $x$ ), it is advantageous to consider both of them simultaneously. Figure 3 is a logarithmic plot of the dipole and (negative) quadrupole moment for a series of shells with $x$ between 1 and 100. The quadratic relationship is evident. The absence of bursts from M31 (see Fig. 1) suggests a maximum sampling distance to shells with $x_{\mathrm{M} 31} \sim 80$, but this is not a well-defined cutoff because the observed angular distribution depends on the actual "occupation numbers" of shells. Of course, the observations clearly suggest small weights for shells with large $\mathrm{x}$ values. Also note, that the $1 \sigma(2 \sigma)$ error contours for isotropy are crossed at $x \sim 10(40)$, which implies that typical BATSE sampling distances are of order $100 \mathrm{kpc}$ for these EGH models. Any equally weighted combination of two shells would create a $(D, Q)$ point somewhere along a curved path shown in the figure for the $(x 1, x 2)=(1100)$ case. Summing many shells over some dynamic range $(x 1, x 2)$ would yield a point inside the segment shown in the figure. For 447 events the one and two sigma statistical errors expected for an isotropic distribution would be consistent with an extended halo that includes observable sources out to $\sim 200 \mathrm{kpc}(1 \sigma)$ or $\sim 100 \mathrm{kpc}(2 \sigma)$. Although the BATSE point itself (cross in Fig. 3 ) can not be reached by these simple EGH models (unless one allows a much larger dynamic range or an additional contribution from a second population), it is clear that EGH models can be consistent with the degree of isotropy implied by BATSE data. The figure also demonstrates the importance of considering both moments simultaneously to constrain EGH models. One can proceed by integrating over shells using a parametrized radial density profile (Wasserman 1992; Hakkila et al. 1992a, b), but

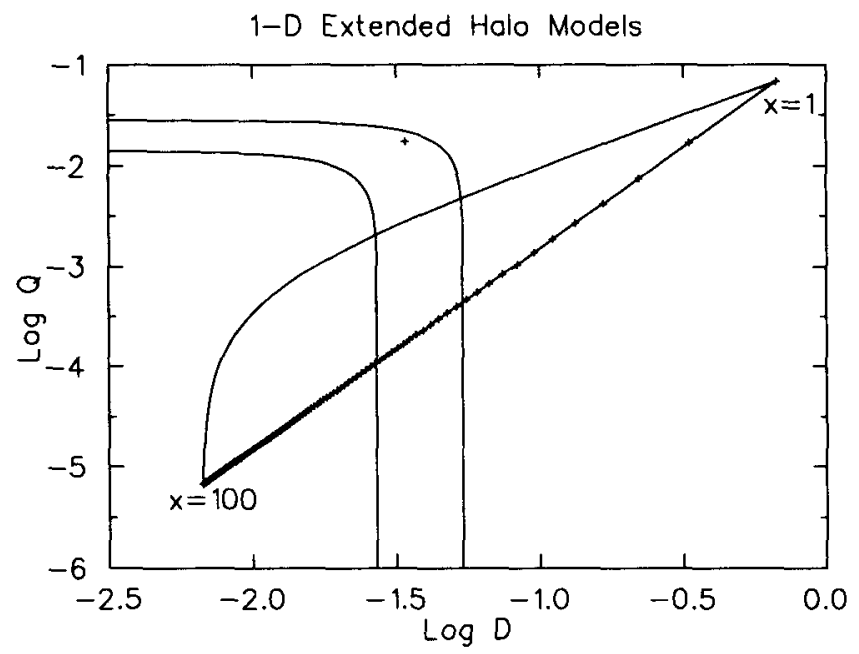

FIG. 3.-Crosses mark the dipole (D) and quadrupole (Q) moments of single shells with radii between $x=1$ and $x=100$. A linear combination of any two shells yields curves like the one shown for the (1100) combination. One and two sigma statistical deviations from isotropy are shown for 447 bursts. The BATSE average (cross) is located close to the $2 \sigma$ contour. 
that constrains only particular models. The geometric properties of EGH models show inherent relations between observable moments that provide powerful constraints, independent of the specifics of how many burst events originate on the various shells.

\section{DISCUSSION AND CONCLUSIONS}

We have argued that the ratio of the number of halo to disk neutron stars could be much less than unity, so that the absence of a disk "contamination" in the BATSE data either argues against the model considered here, or suggests that neutron stars in the halo are much more prolific bursters. The total event rate observed with BATSE is $\sim 10^{3}$ per year, implying a recurrence time of less than $\sim 100$ yr for an estimated $10^{5}$ halo objects. If a typical burst fluence is $10^{-6} \mathrm{ergs} \mathrm{cm}^{-2}$, this implies a lifetime storage requirement of $\sim 10^{49}$ ergs. If this energy is provided by accretion or thermonuclear explosions, less than $\sim 10^{-5} M_{\odot}$ have to be stored in orbit around the neutron star. The low metallicity of Population II objects could be responsible for a much higher efficiency of fall-back of material onto the neutron star (Woosley 1993), leading to the build-up of a planetesimal accretion disk. Lin, Woosley, \& Bodenheimer (1991) estimate that the total mass of the disk is $\sim 10^{-5} M_{\odot}$ and that the mass range of planetesimals is $10^{-12} M_{\odot}$ to $10^{-6}$ $M_{\odot}$. With these parameters one can match rate, brightness-, and sky distribution of GRBs. Slowly accreting neutron stars in the halo undergoing rare Rayleigh-Taylor instabilities due to pycnonuclear reactions do not satisfy the energy requirements (Blaes et al. 1990), unless neutron stars formed in the halo have higher energy storage capabilities.

The observed average $\gamma$-ray burst flux $\left(10^{-6} \mathrm{ergs}^{-2} \mathrm{~cm}^{-1}\right)$ and duration $(\sim 10 \mathrm{~s})$ imply, assuming isotropic emission, an energy release of $\sim 10^{36}$ ergs if the distance scale is $100 \mathrm{pc}$. EGH models increase that scale by a factor $10^{3}$, and cosmological models by more than a factor $10^{6}$. Thus, the energies for EGH or cosmological models are $\sim 10^{42}$ and $10^{48}$ ergs, respectively. The observed variability of $\gamma$-ray burst light curves suggests a small volume, comparable to the size of neutron stars, for the delivery (impulsive or steady over the short duration of the burst ) of this energy. Even if this amount of energy is delivered as pure radiation, two photon pair production $(\gamma \gamma \rightarrow$ $\left.e^{-} e^{+}\right)$will create a pair plasma on timescales that are short in comparison with burst durations. If no baryonic matter is present in the deposition region a pair fireball will be created at temperatures of $\sim 100 \mathrm{keV}$, for EGH distances, or $\sim 100 \mathrm{MeV}$ for cosmological distances (e.g., Piran \& Shemi 1993). For EGH models the Compton optical depth exceeds $10^{9}$, so that regardless of the energy injection mechanism a thermal pair fireball is created. The dynamic expansion of such systems has been studied by many authors (e.g., Goodman 1986; Paczyński 1986; Shemi \& Piran 1990; Meśzáros \& Rees 1992, 1994; Rees \& Meśzáros 1992). The resulting bulk motion of these expanding pair fluids lead to Lorentz factors $(\Gamma)$ of order .0 for EGH and much larger in cosmological models (e.g., ?iran \& Shemi 1993). While these factors are sufficient to Doppler boost the thermal spectrum at $\sim 100 \mathrm{keV}$ into the $\gamma$ oand, they do not allow a significant reduction in the $\gamma \gamma$ opac- ity due to relativistic effects (Krolik \& Pier 1991), so that the spectrum would still be approximately thermal, which is in conflict with the observations. Just as for cosmological models, fireballs at EGH distances are likely to face the problem of "baryonic pollution," which removes burst energy from the photons and transfers it to bulk motion, thus quenching the $\gamma$-ray signal to zero. A possible solution to this problem was suggested by Meśzaŕos \& Rees ( 1993), who considered the late time interaction of the expanding fireball with matter in the burst environment. The resulting shock deceleration of the expanding fireball can extract a significant fraction of the kinetic energy in the bulk flow to generate the observed $\gamma$-ray signal. The low $\Gamma$ values for $E G H$ models remove this possibility of a delayed $\gamma$-ray burst from fireballs in the extended halo. It is thus hard to see how one can actually construct a physical burst model in the EGH that yields not only the right fluxes, time scales, and angular distribution, but also the correct spectra. While particle acceleration mechanisms and spectrum formation processes for bursts from nearby neutron stars are well studied (e.g., Harding 1991; Smith \& Epstein 1993), although not well understood, much work needs to be done to see whether it will be possible to explain $\gamma$-ray bursts in the context of pair fireballs at either EGH or cosmological distances.

We have argued that based on the statistical properties of $\gamma$-ray bursts a Galactic neutron star origin does not have to be abandoned. Because disk distributions do not satisfy the observational constraints, one is forced to consider very extended halos, perhaps with a small contamination by anisotropic sources that have an enhanced quadrupole moment relative to their dipole moment ( see Fig. 3). The observed angular distribution suggests that such halos are more easily created by injection from a preexisting halo (either during Galaxy formation or an ongoing process). Neutrino-, $\gamma$-ray, and nucleosynthesis limits suggest that the halo/disk ratio of neutron stars is small, so that the observed isotropy implies enhanced bursting activity for halo stars and/or suppressed activity for disk stars. Injection of high-velocity neutron stars from the disk can also generate acceptable distribution models, but require that selection effects reduce burst detectability while these stars are still close to the Galactic plane. Cosmological scenarios predict that continued GRB observations should show an increasing degree of isotropy. On the other hand, the EGH model predicts a small but measurable anisotropy. Current BATSE data are consistent with the EGH hypothesis and also with a cosmological origin of $\gamma$-ray bursts (at about the same level of significance). None of the standard pictures for burst generation on or near neutron stars works well for distances much in excess of $100 \mathrm{pc}$. In view of the fact that such a local neutron star origin of $\gamma$-ray bursts is now ruled out by BATSE data, we look for physical processes that operate at larger distances. Many scenarios have been proposed, but it is clear that whether we finally obtain evidence for an EGH or a cosmological origin of $\gamma$-ray bursts, these huge explosions in space are likely to bring us new insights into physics of matter under extreme conditions.

This work was supported in part by NASA grant NAG 5-1578. 


\section{REFERENCES}

Band, D. 1992, ApJ, 400, L63

Blaes, O., Blandford, R., Madau, P., \& Koonin, S. 1990, ApJ, 363, 612

Brainerd, J. J. 1992, Nature, 355, 522

Brock, M. N., Meegan, C. A., Fishman, G. J., Wilson, R. B., Paciesas, W. S., \& Pendleton, G. N. 1992, in Gamma-Ray Bursts ed. W. S. Paciesas \& G. J. Fishman (New York: AIP), 399

Duncan, R. C., Li, H., \& Thompson, C. 1993, in AIP Proc. No. 280 , Compton Gamma-Ray Observatory, ed. M. Friedlander, N. Gehrels, \& D. J. Macomb (New York: AIP), 1074

Duncan, R. C., \& Thompson, C. 1992, ApJ, 392, L9

Eichler, D., \& Silk, J. 1992, Science, 257, 937

Fabian, A. C., \& Podsiadlowski, 1993, MNRAS, 263, 49

Goodman, J. 1986, ApJ, 308, L47

Hakkila, J. \& Meegan, C. A., 1992a, in Gamma-Ray Bursts, ed. W. S. Paciesas, \& G. J. Fishman (New York: AIP), 120

Hakkila, J., et al. 1992b, in AIP Conf. Proc. 280, Compton Gamma-Ray Observatory, ed. M. Friedlander, N. Gehrels, \& D. J. Macomb (New York: AIP), 704

Harding, A. 1991, Phys. Rep., 206, 328

Harrison, P. A., Lyne, A. G., \& Anderson, B. 1993, MNRAS, 261, 113

Hartmann, D. H. 1992, Comm. Astrophys., 16, 231

Hartmann, D. H., \& Epstein, R. 1. 1989, ApJ, 346, 960

Hartmann, D. H., \& The, L.-S. 1993, Ap\&SS, 201, 347

Hartmann, D. H., The, L.-S., Clayton, D. D., Schnepf, N. G., \& Linder, E. V. 1992, in Gamma-Ray Bursts, ed. W. S. Paciesas, \& G. J. Fishman (New York: AIP), 120
Horack, J. M., Meegan, C. A., Fishman, G. J., Wilson, R. B., Paciesas, W. S., Emslie, A. G., Pendleton, G. N., \& Brock, M. N. 1993, ApJ, 413, 293

Krolik, J. H., \& Pier, E. A. 1991, ApJ, 373, 277

Li, H., \& Dermer, C. D. 1992, Nature, 359, 514

Lin, D. N. C., Woosley, S. E., \& Bodenheimer, P. 1991, Nature, 353, 827

Mao, S., \& Paczyński, B. 1992, ApJl, 389, L13

Meegan, C. A., et al. 1992a, Nature, 355, 143

1992b, in AIP Conf. Proc. 26S, Proc. Huntsville Gamma-Ray Burst Workshop, ed. W. S. Paciesas \& G. J. Fishman (New York: AIP), 61

1992c, data supplied to the CGRO public access database 1992d, IAU Circ., No. 5641

Mészáros, P., \& Rees, M. J. 1992, MNRAS, 257, 29P 1994, ApJ, submitted

Paczyński, B. 1986, ApJ, 308, L51 1990, ApJ, 348, 485

1991, Acta Astr., 41, 157

Petrosian, V. 1992, ApJ, 402, L33

Piran, T., \& Shemi, A. 1993, ApJ, 403, L67

Rees, M. J., \& Mészáros, P. 1992, MNRAS, 258, 41P

Shemi, A., \& Piran, T. 1990, ApJ, 365, L55

Shklovskii, I. S., \& Mitrofanov, I. G. 1985, MNRAS, 212, 545

Smith, I. A., \& Epstein, R. I. 1993, ApJ, 410, 315

Wasserman, I. 1992, ApJ, 394, 565

Woosley, S. E. 1993, PASP, in press 